\title{
Rice Diversity Panel Evaluated for Agro-Morphological Diversity by Multivariate Analysis
}

\author{
N. Vishnu Varthini ${ }^{1 *}$, D. Sudhakar ${ }^{2}$, M. Raveendran ${ }^{2}$, S. Rajeswari ${ }^{1}$, \\ S. Manonmani ${ }^{1}$, Shalini Tannidi ${ }^{1}$, P. Balaji Aravindhan ${ }^{1}$, \\ Govindaraj Ponniah ${ }^{1}$, Karthika Gunasekaran ${ }^{1}$ and S. Robin ${ }^{1}$
}

\author{
${ }^{1}$ Centre for Plant Breeding and genetics, ${ }^{2}$ Centre for Plant Molecular Biology and \\ Biotechnology, Tamil Nadu Agricultural University, Coimbatore, Tamil Nadu, India \\ *Corresponding author
}

A B S T R A C T

Genetic diversity assessment for agro morphological traits in a population can be estimated by different methods such as univariate and multivariate analysis. Multivariate analysis is utilized for analyzing more than one variable at once. A diversed collection of 192

Keywords

Rice, Genetic

variation, Agro

morphological traits,

Multivariate analysis,

UPGMA, Principal

component analysis,

Canonical vector

analysis.

Article Info

Accepted:

28 September 2017

Available Online:

10 November 2017 genotypes with traditional landraces and exotic genotypes from 12 countries was evaluated for 12 agro- morphological traits by multivariate analysis which reveals the pattern of genetic diversity and relationship among individuals. Twelve quantitative characters i.e. plant height, leaf length, number of productive tillers, panicle length, number of filled grains, spikelet fertility, days to $50 \%$ flowering; days to harvest maturity, grain length, grain width, grain length width ratio, and single plant yield were measured. Multivariate techniques such as UPGMA cluster analysis, principal component analysis and canonical vector analysis was utilized to examine the variation and to estimate the relative contribution of various traits for total variability. Analysis by UPGMA method had clustered 192 genotypes into seven clusters. Principal component analysis had shown the genetic diversity of the population panel. The cumulative variance of $80.56 \%$ of total variation among 12 characters was explained by the first five axes. Canonical discriminant analysis indicated that the first two functions accounted for more than $86 \%$ of total variance and the traits such as days to $50 \%$ flowering, maturity, grain characters, panicle length and plant height were identified as principal discriminatory characters. These analyses have indicated the presence of variation in the population panel which can be utilized for various crop improvement programs.

\section{Introduction}

Rice is an indispensable staple food for half of the world's population. In countries where rice is used as staple food, the per capita consumption is very high ranging from 62 to $190 \mathrm{~kg} /$ year (Kaiyang et al., 2008). It has the second largest production after wheat with over 503 million tonnes recorded in 2013. While the demand for rice is rising up steadily with steep increase in human population, the land area available for rice production is shrinking due to rapid urbanization and changing life style. New rice cultivars that combine high yield potential, resistance to both biotic and abiotic stress and good grain quality are urgently needed to meet future consumer demands. 
Genetic diversity represents the heritable variation within and between populations of organisms. The success of plant breeding depends on the availability of genetic variation, knowledge about desired traits, and efficient selection strategies that make it possible to exploit existing genetic resource. The pool of genetic variation within an intermating population is the basis for selection as well as for plant improvement.

Before exploiting a population for trait improvement, it is necessary to understand the magnitude of variability in the population which is fundamental for genetic improvement in all crop species. To develop segregating population, genetic distance estimates form the basis for selecting parental combinations with sufficient genetic diversity and for classifying germplasm into heterotic groups for hybrid crop breeding. Population Grouping can be based on geographical origin, agro-morphological traits, pedigree information, or molecular marker data (Liakat Ali et al., 2011).

Genetic distance estimates for population grouping can be estimated by different methods as it is crucial to understand the usable variability existing in the population panel. One of the approaches is to apply multivariate analysis. Cluster analysis can group cultivars and meaningful information of genetic distance between genotypes and clusters can be obtained. Genotypically distant parents are able to produce higher heterosis (Mian, 1989; Ghaderi et al., 1979). It is assumed that the maximum amount of heterosis is manifested in cross combination involving genotypes from the most divergent cluster (Firoz et al., 2008).

Statistical method of classification is usually by multivariate methods as it has extensive use in summarizing and describing the inherent variation among crop genotypes.
Multivariate statistical tools include principal component analysis (PCA), Cluster analysis and discriminate analysis (Oyelola, 2004).

Principal component analysis (PCA) can be used to uncover similarities between variable and classify the cases (genotypes), while cluster analysis on the other hand is concerned with classifying previously unclassified materials (Kaufman and Rouseeuw, 2009). Canonical discriminant analyses were used to determine the relative contribution and linear associations among the traits.

It can separate among-population effects from within population effects by maximizing discrimination among populations when tested against the variation within populations (Riggs, 1973; Tai, 1989).

Multivariate analysis has been used in various crops i.e., Rice (Sanni et al., 2012, Chakravorthy et al., 2013), soybean (Bhawana Sharma and Brijvirsingh, 2012), coconut (Odewale et al., 2012), safflower, sorghum and oil palm to study the pattern of variation. The study aimed to determine level of germplasm variation and identify and classify variation for grouping the accessions by taking into account several characteristics and relationship between them.

\section{Materials and Methods}

\section{Experimental material}

The germplasm collection consisting of 192 rice accessions was used in this study, which consist of land races and varieties collected from nine different states of India as well as from different countries (Table 1). It has 82 landraces from different agro climatic zones of Tamil Nadu and two landraces from Orissa. 62 exotic genotypes from Argentina, Bangladesh, Brazil, Bulgaria, China, 
Colombia, Indonesia, Philippines, Taiwan, Uruguay, Venezuela and United States and 46 varieties and improved genotypes from different states of India constitute the population panel of 192 genotypes. For easy identification and retrieval, each accession was named as RG 1 to RG 192.

\section{Experimental site}

A set of 192 genotypes were grown in Paddy Breeding Station, Department of Rice, Tamil Nadu Agricultural University, India during Rabi 2013. This area is situated at latitude of $11^{\circ} \mathrm{N}$ and longitude of $77^{\circ} \mathrm{E}$ with clayey soil of $\mathrm{pH} 7.8$.

\section{Methods}

One hundred and ninety two genotypes were transplanted 21 days after sowing as two seedlings per hill in randomized complete block design with a spacing of $20 \times 20 \mathrm{~cm}$.

Each plot per accession consisted of four rows each 0.8 by $3.6 \mathrm{~m}$ long at a distance of $40 \mathrm{~cm}$ between the plots. Normal cultural practices were followed as per standard recommendation.

Twelve quantitative characters were measured according to methods in the descriptors for rice O. sativa (IRRI, 1980). Variables considered in the descriptive and multivariate analyses were morphological (plant height, leaf length, number of productive tillers, panicle length, number of filled grains, spikelet fertility), phenological (days to 50\% flowering and days to harvest maturity from the day of seeding), and grain traits (grain length, grain width, grain length width ratio, and single plant yield).

\section{Statistical analysis}

The observations recorded on 12 traits were statistically analyzed in SPSS16.0 to cluster the genotypes based on genetic similarity. Unweighted pair group method of average linkage (UPGMA) constructed by SPSS16.0 was used to classify the accessions into clusters. The PCA analysis reduces the dimensions of a multivariate data to a few principal axes, generates an Eigen vector for each axis and produces component scores for the characters (Sneath and Sokal, 1973; Ariyo and Odulaja, 1991). Canonical discriminate analysis measure the axis along which variation between entries were maximum (Rezai and Frey, 1990; Ariyo, 1993).

\section{Results and Discussion}

The maximum, minimum, sum, mean, standard deviation (SD) and coefficient of variation $(\mathrm{CV})$ for the measured traits are presented in table 2 . The largest variation was observed for number of productive tillers with $\mathrm{CV}$ of $28.03 \%$ followed by number of filled grains per panicle $(\mathrm{CV}=27)$, single plant yield (23.19), leaf length (23.02), grain length width ratio (22.16). Days to maturity has shown the least variation with the $\mathrm{CV}$ of $9.74 \%$.

The genotype RG1 has taken the longest days for flowering as well as maturity. The taller genotype is RG20 whereas RG111 has short stature. RG183 has more number of productive tillers but RG164 has higher single plant yield.

Spikelet fertility ranges from $95.7 \%$ in RG131 to 54.2 in RG25. The accession with longest grain was RG57 (10.5) and largest grain width in RG160 (3.7) which is a bold grain type. The slim grain type with lesser grain width was RG95 (1.5) and shortest grain was RG111 (5.8).

\section{Cluster analysis}

Analysis by UPGMA method has clustered 192 genotypes into seven clusters (Table 1). 
Landraces has diffused across the different clusters. $72 \%$ of the landraces (62 landraces) has amalgamated in cluster 2. Cluster 1,3,4,5 and 7 has the remaining landraces. Cluster 1 has two landraces RG1 (Mapillai samba) and RG 106 (Katta samba). Cluster 7 has one landrace RG164 (Thillainayagam). Cluster 3 has 7 landraces (RG4, RG12, RG33, RG42, RG50, RG110andRG120). Nine landraces (RG32， RG73， RG97， RG109， RG155, RG163, RG168, RG179 and RG192) spread across cluster 4 . Cluster 5 has three landraces (RG24, RG25 and RG44).

The population panel has 61 exotic genotypes which has been clustered in group 2 (22 genotypes), group 4(29 genotypes) and each 2 genotypes in cluster 5 and 6 . This panel also has 47 improved genotypes and varieties from different states of India. Majority of the improved genotypes and varieties $(51 \%)$ has clustered in group 4. Remaining improved genotypes and varieties has dispersed in cluster 2 (13 genotypes), cluster 3(8 genotypes), cluster 5(1genotype) and cluster 6 (1 genotype).

\section{Principal component analysis}

Principal component analysis has shown the genetic diversity of the population panel. The cumulative variance of $80.56 \%$ by the first five axes with Eigen value of $>1.0$ (Figure 1 and 2) indicates that the identified traits within the axes exhibited great influence on the phenotype of population panel (Table 3 and 4).

The different morphological traits contribute for total variation calculated for each component. For Component 1 which has the contribution of Days to $50 \%$ flowering (loadings -0.87), leaf length (0.78), plant height (0.765), panicle length (0.637), days to maturity (0.853) and number of filled grains $(0.352)$ for $28.46 \%$ of the total variability.
For component 2, grain width (0.886) and grain length width ratio (0.951) has contributed $16.8 \%$ of total variability. Similarly spikelet fertility (0.771) and single plant yield (0.542), grain length (0.81), number of productive tillers (0.846) has contributed for the total variation of $14.4 \%$, $11.7 \%$ and $9.3 \%$ from component 3 , component 4 and component 5 respectively.

\section{Canonical Discriminant analysis}

Canonical discriminant analysis simultaneously examines the differences in the morphological variables and indicates the relative contribution of each variable to accession discrimination (Vaylay and van Santen, 2002).

Quantitative variables were considered as independent and the clusters identified by cluster analysis as dependent variables. The first four Discriminant functions were statistically significant according to the chisquare test at a probability of 0.01. Proper values and the distribution of their variances indicated that the first two functions accounted for more than $86 \%$ of total variance. Wilks' lambda coefficients for these two functions were precisely the lowest, indicating an almost perfect discrimination regarding the remaining functions. The significant $(p<0.001)$ canonical correlation between the accessions and the first canonical variate (canonical correlation $=0.851$ ) and second canonical variate (canonical correlation $=0.748$ ) indicates that the canonical variates can explain the differentiation of the accessions.

The standardized canonical discriminant coefficients can be used to rank the importance of each variable. A high standardized discriminant function coefficient for a trait might mean that the variable has greater discriminating ability. The first 
canonical Discriminant function is dominated by plant height, days to $50 \%$ flowering and days to maturity (Table 5). Number of filled grains per panicle, panicle length spikelet fertility and grain length contribute for second canonical Discriminant function. It is therefore evident in the canonical discrimination that the composition of the accessions differs chiefly in days to $50 \%$ flowering, maturity, grain characters, panicle length and plant height. Centroids are discriminant score for each group when the variable means (rather than individual values for each case) are entered into the function. The Proximity of group centroids indicates the errors in classification. The distance between group centroids for different clusters is far away which indicates the precision of classification level (Figure 3).

Fig.1 Scattered Diagram of first two components explaining the diversity of genotypes

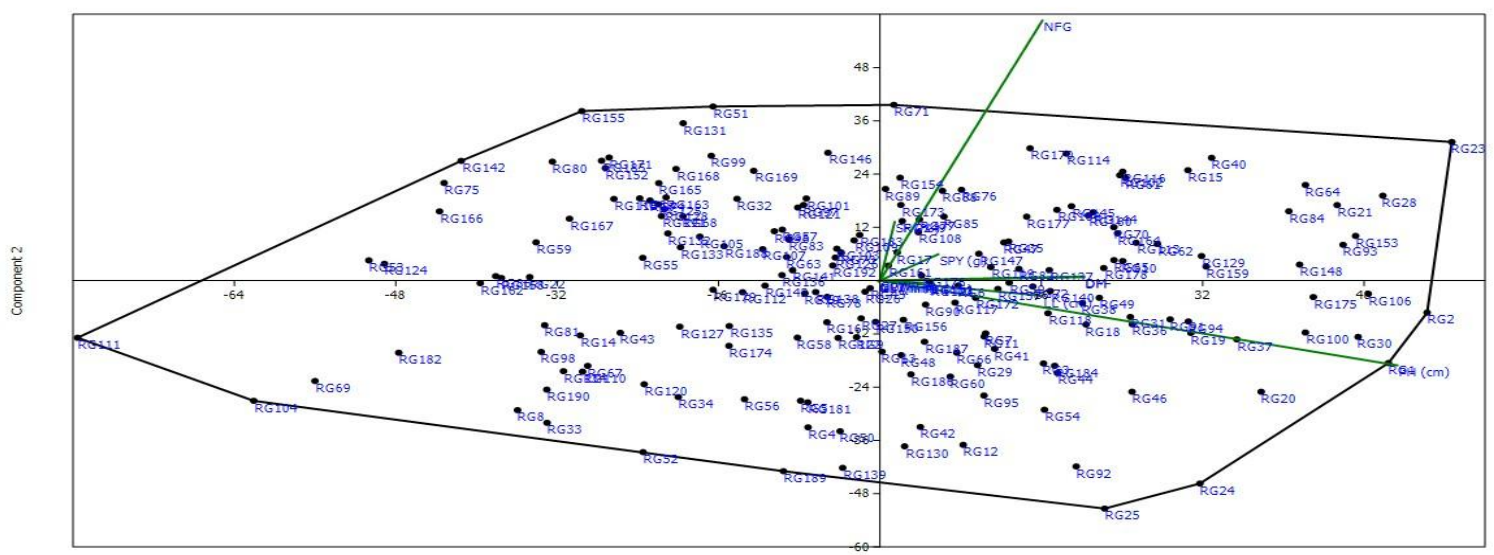

Component 1

Fig.2 Eigen value for the corresponding principal components

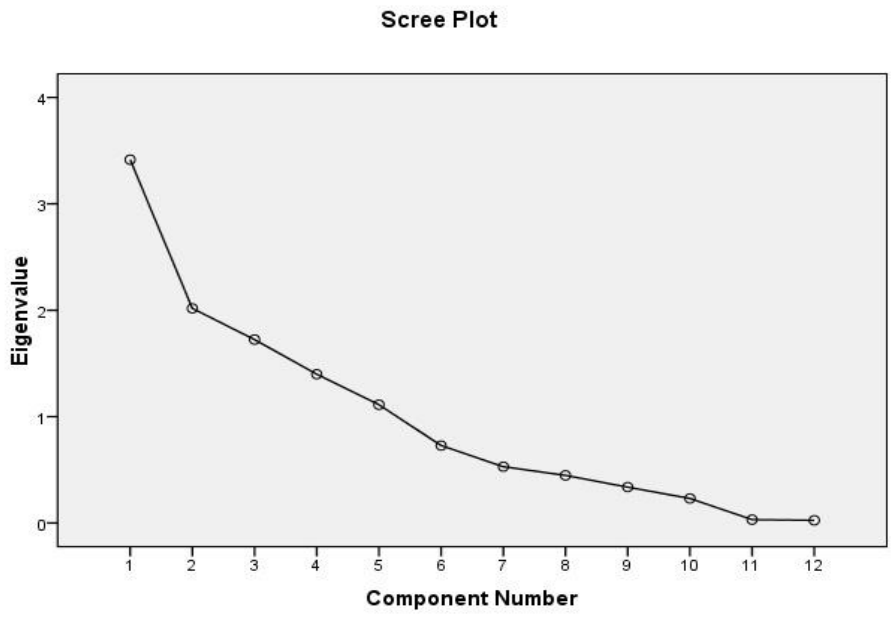


Fig.3 Group centroids for different clusters is far away which indicates the precision of classification level

\section{Canonical Discriminant Functions}
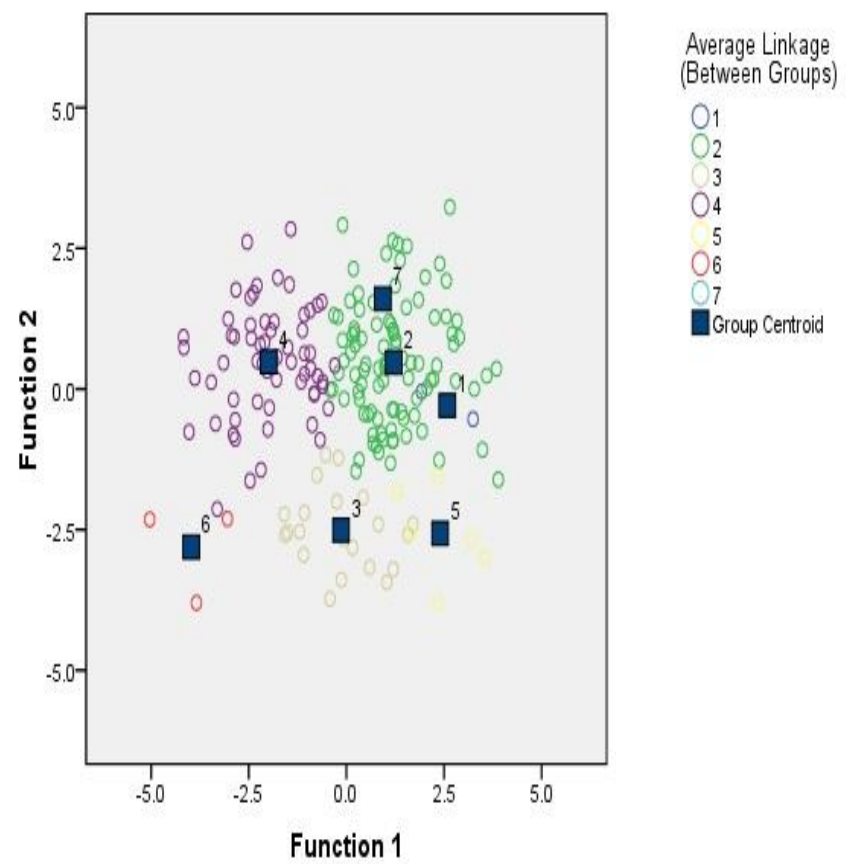

Table.1 Genotypes information with clustering pattern

\begin{tabular}{|l|l|l|l|l|}
\hline G. & Genotypes & Parentage & Origin & Cluste \\
\hline RG1 & Mapillai samba & Landrace & Tamil Nadu, India & 1 \\
\hline RG10 & Katta samba & Landrace & Tamil Nadu, India & 1 \\
\hline RG2 & CK 275 & CO50 X KAVUNI & Tamil Nadu, India & 2 \\
\hline RG3 & Senkar & Landrace & Tamil Nadu, India & 2 \\
\hline RG6 & CHIR 5 & Improved chinsurah & West Bengal & 2 \\
\hline RG7 & Kudaivazhai & Landrace & Tamil Nadu, India & 2 \\
\hline RG9 & Kuruvaikalanjiyam & Landrace & Tamil Nadu, India & 2 \\
\hline RG10 & Nava konmani & Landrace & Tamil Nadu, India & 2 \\
\hline RG11 & CHIR 10 & Improved chinsurah & West Bengal & 2 \\
\hline RG13 & CHIR 2 & Improved chinsurah & West Bengal & 2 \\
\hline RG15 & Palkachaka & Landrace & Tamil Nadu, India & 2 \\
\hline RG16 & Thooyala & Landrace & Tamil Nadu, India & 2 \\
\hline RG17 & Chivapuchithiraikar & Landrace & Tamil Nadu, India & 2 \\
\hline RG18 & CHIR 11 & Improved chinsurah & West Bengal & 2 \\
\hline RG19 & Koolavalai & Landrace & Tamil Nadu, India & 2 \\
\hline RG20 & Kalvalai & Landrace & Tamil Nadu, India & 2 \\
\hline
\end{tabular}




\begin{tabular}{|c|c|c|c|c|}
\hline RG21 & \begin{tabular}{|l} 
Mohini samba \\
\end{tabular} & Landrace & Tamil Nadu, India & 2 \\
\hline RG23 & Koombalai & Landrace & Tamil Nadu, India & 2 \\
\hline RG26 & Rascadam & Landrace & Tamil Nadu, India & 2 \\
\hline RG27 & Muzhikaruppan & Landrace & Tamil Nadu, India & 2 \\
\hline RG28 & Kaatukuthalam & Landrace & Tamil Nadu, India & 2 \\
\hline RG29 & Vellaikattai & Landrace & Tamil Nadu, India & 2 \\
\hline RG30 & Poongar & Landrace & Tamil Nadu, India & 2 \\
\hline RG31 & Chinthamani & Landrace & Tamil Nadu, India & 2 \\
\hline RG35 & CK 143 & CO50 X KAVUNI & Tamil Nadu, India & 2 \\
\hline RG36 & Kattikar & Landrace & Tamil Nadu, India & 2 \\
\hline RG37 & Shenmolagai & Landrace & Tamil Nadu, India & 2 \\
\hline RG38 & Velli samba & Landrace & Tamil Nadu, India & 2 \\
\hline RG39 & Kaatuponni & Landrace & Tamil Nadu, India & 2 \\
\hline RG40 & kakarathan & Landrace & Tamil Nadu, India & 2 \\
\hline RG41 & Godavari samba & Landrace & Tamil Nadu, India & 2 \\
\hline RG45 & RPHP 105 & Moirangphou & MANIPUR & 2 \\
\hline RG47 & Machakantha & Landrace & Orissa, India & 2 \\
\hline RG48 & Kalarkar & Landrace & Tamil Nadu, India & 2 \\
\hline RG49 & Valanchennai & Landrace & Tamil Nadu, India & 2 \\
\hline RG58 & Kodaikuluthan & Landrace & Tamil Nadu, India & 2 \\
\hline RG60 & Rama kuruvaikar & Landrace & Tamil Nadu, India & 2 \\
\hline RG61 & Kallundai & Landrace & Tamil Nadu, India & 2 \\
\hline RG62 & Purple puttu & Landrace & Tamil Nadu, India & 2 \\
\hline RG63 & IG 71(EC 728651- & TEPI BORO::IRGC 27519-1 & IRRI, Philippines & 2 \\
\hline RG64 & Ottadaiyan & Landrace & Tamil Nadu, India & 2 \\
\hline RG65 & IG 56 (EC 728700- & BICO BRANCO & Brazil & 2 \\
\hline RG66 & Jeevan samba & Landrace & Tamil Nadu, India & 2 \\
\hline RG70 & Karthi samba & Landrace & Tamil Nadu, India & 2 \\
\hline RG72 & Aarkadukichili & Landrace & Tamil Nadu, India & 2 \\
\hline RG76 & Mattakuruvai & Landrace & Tamil Nadu, India & 2 \\
\hline RG77 & Karuthakar & Landrace & Tamil Nadu, India & 2 \\
\hline RG78 & RPHP 165 & Tilakkachari & West Bengal & 2 \\
\hline RG79 & Manavari & Landrace & Tamil Nadu, India & 2 \\
\hline RG82 & Thooyamalli & Landrace & Tamil Nadu, India & 2 \\
\hline RG84 & Velsamba & Landrace & Tamil Nadu, India & 2 \\
\hline RG85 & RPHP 104 & Kasturi (IET 8580) & UTTARKHAND & 2 \\
\hline RG88 & Saranga & Landrace & Tamil Nadu, India & 2 \\
\hline RG90 & IG 61(EC 728731- & CRIOLLO LA FRIA & Venezuela & 2 \\
\hline RG91 & IG 23(EC 729391- & MAHA PANNITHI::IRGC 51021- & IRRI, Philippines & 2 \\
\hline RG93 & uppumolagai & Landrace & Tamil Nadu, India & 2 \\
\hline RG94 & Karthigai samba & Landrace & Tamil Nadu, India & 2 \\
\hline RG95 & Jeeraga samba & Landrace & Tamil Nadu, India & 2 \\
\hline RG10 & IG 7(EC 729598- & VARY MAINTY::1RGC 69910-1 & IRRI, Philippines & 2 \\
\hline RG10 & Varakkal & Landrace & Tamil Nadu, India & 2 \\
\hline
\end{tabular}




\begin{tabular}{|c|c|c|c|c|}
\hline RG10 & Mattaikar & Landrace & Tamil Nadu, India & 2 \\
\hline RG10 & Red sirumani & Landrace & Tamil Nadu, India & 2 \\
\hline RG11 & IG 45(EC 728768- & FORTUNA & Puerto Rico & 2 \\
\hline RG11 & RPHP 159 & RadhuniPagal & BANGLADESH & 2 \\
\hline RG11 & RPHP 27 & Azucena & HARYANA & 2 \\
\hline RG11 & IG 65(EC 729024- & GODA HEENAT1::IRGC 31393-1 & IRRI, Philippines & 2 \\
\hline RG11 & Ponmani samba & Landrace & Tamil Nadu, India & 2 \\
\hline RG11 & Ganthasala & Landrace & Tamil Nadu, India & 2 \\
\hline RG12 & Kaliyan samba & Landrace & Tamil Nadu, India & 2 \\
\hline RG12 & IG 2(EC 729808- & BLUEBONNET 50::IRGC 1811-1 & IRRI, Philippines & 2 \\
\hline RG12 & Kallimadayan & Landrace & Tamil Nadu, India & 2 \\
\hline RG12 & IG 38(EC 728742 - & DELREX & UNITED STATES & 2 \\
\hline RG13 & IG 37(EC 728715- & CENIT & ARGENTINA & 2 \\
\hline RG13 & Sigappukuruvikar & Landrace & Tamil Nadu, India & 2 \\
\hline RG14 & Raja mannar & Landrace & Tamil Nadu, India & 2 \\
\hline RG14 & IG 46(IC 471826- & BABER & INDIA & 2 \\
\hline RG14 & Chetty samba & Landrace & Tamil Nadu, India & 2 \\
\hline RG14 & IG 60(EC 728730- & CREOLE & Belize & 2 \\
\hline RG14 & IG 58(EC 728725- & CI 11011 & UNITED STATES & 2 \\
\hline RG14 & Chinnaadukunel & Landrace & Tamil Nadu, India & 2 \\
\hline RG15 & IG 14(IC 517381- & MALACHAN::IRGC 54748-1 & IRRI, Philippines & 2 \\
\hline RG15 & IG 32(EC 728838- & NOVA & United States & 2 \\
\hline RG15 & Sembilipiriyan & Landrace & Tamil Nadu, India & 2 \\
\hline RG15 & IG 12(EC 729626- & SHESTAK::IRGC 32351-1 & IRRI, Philippines & 2 \\
\hline RG15 & Karungan & Landrace & Tamil Nadu, India & 2 \\
\hline RG15 & Sembala & Landrace & Tamil Nadu, India & 2 \\
\hline RG16 & IG 72(EC 728650- & TD 25::IRGC 9146-1 & IRRI, Philippines & 2 \\
\hline RG16 & Panamarasamba & Landrace & Tamil Nadu, India & 2 \\
\hline RG17 & RPHP 42 & Shalimar Rice -1 & JAMMU and & 2 \\
\hline RG17 & IG 25(EC 729728- & LOHAMBITRO 224::GERVEX & IRRI, Philippines & 2 \\
\hline RG17 & IG 73(EC 728627- & MAKALIOKA 34::1RGC 6087-1 & IRRI, Philippines & 2 \\
\hline RG17 & Vellaikudaivazhai & Landrace & Tamil Nadu, India & 2 \\
\hline RG17 & Kodai & Landrace & Tamil Nadu, India & 2 \\
\hline RG17 & Kallundaikar & Landrace & Tamil Nadu, India & 2 \\
\hline RG17 & IG 17(EC 728900- & SIGADIS & INDONESIA & 2 \\
\hline RG18 & IG 59(EC 728729- & COPPOCINA & BULGARIA & 2 \\
\hline RG18 & IG 18(EC 728892- & SERATOES HARI & INDONESIA & 2 \\
\hline RG18 & IG 28(EC 728920- & TIA BURA & INDONESIA & 2 \\
\hline RG18 & Vadakathi samba & Landrace & Tamil Nadu, India & 2 \\
\hline RG4 & Murugankar & Landrace & Tamil Nadu, India & 3 \\
\hline RG5 & CHIR 6 & Improved chinsurah & West Bengal & 3 \\
\hline RG8 & CHIR 8 & Improved chinsurah & West Bengal & 3 \\
\hline RG12 & Vellaichithiraikar & Landrace & Tamil Nadu, India & 3 \\
\hline RG33 & Malayalathan samba & Landrace & Tamil Nadu, India & 3 \\
\hline
\end{tabular}




\begin{tabular}{|c|c|c|c|c|}
\hline RG34 & RPHP 125 & NDR 2026 (RICHA) & UTTAR & 3 \\
\hline RG42 & Earapalli samba & Landrace & Tamil Nadu, India & 3 \\
\hline RG43 & RPHP 129 & Kamad & JAMMU and & 3 \\
\hline RG50 & Sornavari & Landrace & Tamil Nadu, India & 3 \\
\hline RG52 & ARB 58 & Variety & Karnataka & 3 \\
\hline RG56 & RPHP 59 & Taroari Basmati/karnal local & HARYANA & 3 \\
\hline RG67 & RPHP 106 & akutphou & MANIPUR & 3 \\
\hline RG11 & Norungan & Landrace & Tamil Nadu, India & 3 \\
\hline RG12 & Thattan samba & Landrace & Tamil Nadu, India & 3 \\
\hline RG12 & IG 10(EC 729686- & HASAN SERALIRGC 79564-C1 & IRRI, Philippines & 3 \\
\hline RG13 & IG 39(EC 728779- & HONDURAS & HONDURAS & 3 \\
\hline RG13 & IG 9(EC 729682- & GEMJYA JYANAM::IRGC & IRRI, Philippines & 3 \\
\hline RG13 & RPHP 138 & EDAVANKUDI POKKALI & Kerala, India & 3 \\
\hline RG18 & IG 52(EC 728756- & DOURADO AGULHA & BRAZIL & 3 \\
\hline RG18 & IG 41(EC 728800- & KANIRANGA & Indonesia & 3 \\
\hline RG19 & IG 26(IC 0590943- & BASMATI 370::IRGC 3750-1 & IRRI, Philippines & 3 \\
\hline RG14 & Jothi & variety & Kerala, India & 4 \\
\hline RG22 & IR 36 & IR 1561 X IR 24 X Oryzanivara X & IRRI, Philippines & 4 \\
\hline RG32 & Thogai samba & Landrace & Tamil Nadu, India & 4 \\
\hline RG51 & RPHP 134 & NJAVARA & Kerala & 4 \\
\hline RG53 & IR 68144-2B-2-2-3-1- & IR 72 X ZAWA BONDAY & IRRI, Philippines & 4 \\
\hline RG55 & IG 67(EC 729050- & IR 77384-12-35-3-12-1-B::IRGC & IRRI, Philippines & 4 \\
\hline RG57 & RPHP 103 & Pant sugandhdhan -17 & UTTARKHAND & 4 \\
\hline RG59 & RPHP 68 & Subhdra & Orissa, India & 4 \\
\hline RG68 & IG 63(EC 728711- & CAAWA/FORTUNA & IRRI, Philippines & 4 \\
\hline RG71 & IG 27(IC 0590934- & ARC 11345::IRGC 21336-1 & IRRI, Philippines & 4 \\
\hline RG73 & Kunthali & Landrace & Tamil Nadu, India & 4 \\
\hline RG74 & ARB 65 & Variety & Karnataka & 4 \\
\hline RG75 & IG 21(EC 729334- & HONGJEONG::IRGC 73052-1 & IRRI, Philippines & 4 \\
\hline RG80 & IG 66 (EC 729047- & IR 71137-243-2-2-3-3::IRGC & IRRI, Philippines & 4 \\
\hline RG81 & CB-07-701-252 & Improved line & Tamil Nadu, India & 4 \\
\hline RG83 & RPHP 93 & Type-3 (Dehradooni Basmati) & UTTARKHAND & 4 \\
\hline RG86 & RPHP 102 & Kanchana & Kerala, India & 4 \\
\hline RG87 & IG 40 (EC 728740- & DEE GEO WOO GEN & TAIWAN & 4 \\
\hline RG89 & IR 83294-66-2-2-3-2 & DAESANBYEO X IR65564-44-5- & IRRI, Philippines & 4 \\
\hline RG96 & RP-BIO-226 & IMPROVED SAMBHA & ANDHRA & 4 \\
\hline RG97 & Varigarudan samba & Landrace & Tamil Nadu, India & 4 \\
\hline RG98 & IG 5(EC 729642- & IR 65907-116-1-B::C1 & IRRI, Philippines & 4 \\
\hline RG99 & IG 31(EC 728844- & ORYZICA LLANOS 5 & Colombia & 4 \\
\hline RG10 & RPHP 52 & SEBATI & Orissa, India & 4 \\
\hline RG10 & IG 6(EC 729592- & SOM CAU 70 A::1RGC 8227-1 & IRRI, Philippines & 4 \\
\hline RG10 & RH2-SM-1-2-1 & SWARNA X MOROBERAKAN & Tamil Nadu, India & 4 \\
\hline RG10 & Vadivel & Landrace & Tamil Nadu, India & 4 \\
\hline RG11 & IG 35(EC 728858- & PATE BLANC MN 1 & Cote D'Ivoire & 4 \\
\hline
\end{tabular}




\begin{tabular}{|c|c|c|c|c|}
\hline RG11 & IG 43(EC 728788- & IR-44595 & IRRI, Philippines & 4 \\
\hline RG12 & IG 74(EC 728622- & KINANDANG PATONG::IRGC & IRRI, Philippines & 4 \\
\hline RG12 & IG 29(EC 728925- & TOX 782-20-1 & NIGERIA & 4 \\
\hline RG12 & RPHP 55 & Kalinga -3 & Orissa & 4 \\
\hline RG12 & IG 75(EC 728587- & AEDAL::IRGC 55441-1 & IRRI, Philippines & 4 \\
\hline RG13 & RPHP 90 & $182(\mathrm{M})$ & Andhra Pradesh & 4 \\
\hline RG13 & IG 33(EC 728938- & WC 3397 & JAMAICA & 4 \\
\hline RG13 & IG 42(EC 728798- & KALUBALA VEE & SRILANKA & 4 \\
\hline RG13 & RPHP 161 & ChampaKhushi & & 4 \\
\hline RG13 & IG 8(EC 729601- & XI YOU ZHAN::1RGC 78574-1 & IRRI, Philippines & 4 \\
\hline RG14 & IG 44(EC 728762- & EDITH & UNITED STATES & 4 \\
\hline RG14 & Sasyasree & TKM 6 × IR 8 & West Bengal & 4 \\
\hline RG14 & IR 75862-206 & IR 75083 X IR $65600-81-5-3-2$ & IRRI, Philippines & 4 \\
\hline RG14 & RH2-SM-2-23 & SWARNA X MOROBERAKAN & Tamil Nadu, India & 4 \\
\hline RG15 & RPHP 47 & Pathara (CO-18 x Hema) & India & 4 \\
\hline RG15 & IG 48(EC 729203- & DINOLORES::IRGC 67431-1 & IRRI, Philippines & 4 \\
\hline RG15 & Sonamahsuri & Landrace & Tamil Nadu, India & 4 \\
\hline RG15 & IG 13(EC 729640- & CURINCA::C1 & IRRI, Philippines & 4 \\
\hline RG16 & IR 64 & IR-5857-33-2-1 x IR-2061-465-1- & IRRI, Philippines & 4 \\
\hline RG16 & Mikuruvai & Landrace & Tamil Nadu, India & 4 \\
\hline RG16 & ARB 64 & Variety & Karnataka & 4 \\
\hline RG16 & RPHP 140 & VYTILLA ANAKONDAN & Kerala & 4 \\
\hline RG16 & IG 70(EC 729045- & IR43::1RGC 117005-1 & IRRI, Philippines & 4 \\
\hline RG16 & Haladichudi & Landrace & Orissa, India & 4 \\
\hline RG16 & IG 24(EC 728751- & DNJ 140 & BANGLADESH & 4 \\
\hline RG17 & RPHP 44 & BR- 2655 & KARNATAKA & 4 \\
\hline RG17 & IG 51(EC 728772- & GOGO LEMPUK & Indonesia & 4 \\
\hline RG17 & Avasara samba & Landrace & Tamil Nadu, India & 4 \\
\hline RG18 & ARB 59 & Variety & Karnataka & 4 \\
\hline RG18 & RPHP 163 & Seeta sail & West Bengal & 4 \\
\hline RG18 & RPHP 36 & TKM-9 & Tamil Nadu, India & 4 \\
\hline RG18 & RPHP 80 & $24(\mathrm{~K})$ & Andhra Pradesh & 4 \\
\hline RG19 & IG 15(EC 728910- & SZE GUEN ZIM & CHINA & 4 \\
\hline RG19 & Nootripathu & Landrace & Tamil Nadu, India & 4 \\
\hline RG24 & Tadukan & Landrace & Tamil Nadu, India & 5 \\
\hline RG25 & Sornakuruvai & Landrace & Tamil Nadu, India & 5 \\
\hline RG44 & Mangam samba & Landrace & Tamil Nadu, India & 5 \\
\hline RG46 & IG 4 (EC 729639- & TD2: :IRGC 9148-1 & IRRI, Philippines & 5 \\
\hline RG54 & PTB 19 & Variety & Kerala, India & 5 \\
\hline RG92 & IG 49(EC 729102- & MENAKELY ::IRGC 69963-1 & IRRI, Philippines & 5 \\
\hline RG69 & RPHP 48 & Bindli & UTTARKHAND & 6 \\
\hline RG10 & IG 53(EC 728752- & CAROLINA RINALDO & URUGUAY & 6 \\
\hline RG11 & IG 20(EC 729293- & CHIGYUNGDO::IRGC 55466-1 & IRRI, Philippines & 6 \\
\hline RG16 & Thillainayagam & Landrace & Tamil Nadu, India & 7 \\
\hline
\end{tabular}


Table.2 Characteristic means and variations of 192 accessions panel

\begin{tabular}{|c|c|c|c|c|c|c|c|c|}
\hline \multirow{2}{*}{ Variable } & \multirow[b]{2}{*}{ Sum } & \multirow[b]{2}{*}{ Mean } & \multirow[b]{2}{*}{ SD } & \multirow[b]{2}{*}{$\mathrm{CV}$} & \multicolumn{2}{|l|}{ MIN } & \multicolumn{2}{|l|}{ MAX } \\
\hline & & & & & Value & Accessions & Value & Accessions \\
\hline DFF & 16380.1 & 85.31 & 11.07 & 12.97 & 66.0 & RG69 & 123.0 & RG1 \\
\hline $\mathrm{LL}(\mathrm{cm})$ & 7227.7 & 37.64 & 8.67 & 23.02 & 20.8 & RG104 & 62.4 & RG106 \\
\hline $\mathrm{PH}(\mathrm{cm})$ & 22231.6 & 115.79 & 21.79 & 18.82 & 51.0 & RG111 & 162.3 & RG20 \\
\hline NPT & 2547.95 & 13.27 & 3.72 & 28.03 & 5.0 & RG147 & 26.5 & RG183 \\
\hline PL (cm) & 4237.2 & 22.07 & 2.74 & 12.42 & 11.6 & RG111 & 31.1 & RG85 \\
\hline $\mathrm{DM}$ & 22556.5 & 117.48 & 11.44 & 9.74 & 94.0 & RG43, RG59 & 155.0 & RG1 \\
\hline NFG & 13201.1 & 68.76 & 18.56 & 27.00 & 26.6 & RG189 & 112.0 & RG23 \\
\hline SF (\%) & 15907.25 & 82.85 & 6.93 & 8.37 & 54.2 & RG25 & 95.7 & RG131 \\
\hline $\mathrm{GL}(\mathrm{mm})$ & 1592.5 & 8.29 & 0.89 & 10.68 & 5.8 & RG111 & 10.5 & RG57 \\
\hline $\mathrm{GW}(\mathrm{mm})$ & 483.785 & 2.52 & 0.49 & 19.49 & 1.5 & RG95 & 3.7 & RG160 \\
\hline GLWR & 661.4 & 3.44 & 0.76 & 22.16 & 1.8 & RG122 & 5.6 & RG47 \\
\hline SPY (g) & 4832.88 & 25.17 & 5.84 & 23.19 & 12.0 & RG104 & 55.5 & RG164 \\
\hline
\end{tabular}

Table.3 Eigen value and percent of total variation and component matrix for the principal component axes

\begin{tabular}{|l|l|l|l|l|l|}
\hline PC & 1 & 2 & 3 & 4 & 5 \\
\hline Eigen values & 3.415 & 2.017 & 1.724 & 1.399 & 1.112 \\
\hline \% of Variance & 28.459 & 16.810 & 14.369 & 11.658 & 9.267 \\
\hline Cumulative \% & 28.459 & 45.269 & 59.638 & 71.296 & 80.563 \\
\hline Component Matrix & $\mathbf{0 . 8 7}$ & 0.047 & -0.222 & -0.276 & -0.125 \\
\hline DFF & $\mathbf{0 . 7 8}$ & -0.297 & -0.062 & -0.063 & 0.108 \\
\hline LL $(\mathrm{cm})$ & $\mathbf{0 . 7 6 5}$ & -0.11 & -0.025 & 0.267 & 0.214 \\
\hline PH $(\mathrm{cm})$ & -0.056 & 0.179 & 0.284 & -0.194 & $\mathbf{0 . 8 4 6}$ \\
\hline NPT & $\mathbf{0 . 6 3 7}$ & 0.134 & -0.084 & 0.524 & -0.029 \\
\hline PL $(\mathrm{cm})$ & $\mathbf{0 . 8 5 3}$ & 0.034 & -0.219 & -0.279 & -0.116 \\
\hline DM & $\mathbf{0 . 3 5 2}$ & 0.258 & 0.711 & 0.003 & -0.326 \\
\hline NFG & 0.03 & 0.233 & $\mathbf{0 . 7 7 1}$ & -0.003 & -0.294 \\
\hline SF $(\%)$ & -0.147 & 0.232 & -0.256 & $\mathbf{0 . 8 1}$ & -0.031 \\
\hline GL $(\mathrm{mm})$ & -0.089 & $\mathbf{- 0 . 8 8 6}$ & 0.154 & 0.323 & -0.047 \\
\hline GW $(\mathrm{mm})$ & 0.024 & $\mathbf{0 . 9 5 1}$ & -0.228 & 0.098 & 0.034 \\
\hline GLWR & 0.416 & 0.012 & $\mathbf{0 . 5 4 2}$ & 0.295 & 0.335 \\
\hline SPY $(\mathrm{g})$ &
\end{tabular}

Table.4 Discriminant functions that distinguish between clusters of rice accessions

\begin{tabular}{|c|c|c|c|c|c|c|c|c|}
\hline & & Variance \% & & & & & & \\
\hline Function & Eigen Value & Proportion & Cumulative & Canonical & Wilks' & Chi- & $\overline{d f}$ & Sig. \\
\hline 1 & 2.622 & 58.4 & 58.4 & 0.851 & 0.071 & 480.035 & 72 & $<0.0001$ \\
\hline 2 & 1.266 & 28.2 & 86.7 & 0.748 & 0.257 & 246.429 & 55 & $<0.0001$ \\
\hline 3 & 0.295 & 6.6 & 93.2 & 0.477 & 0.583 & 97.926 & 40 & $<0.0001$ \\
\hline 4 & 0.213 & 4.7 & 98 & 0.419 & 0.755 & 51.029 & 27 & 0.003 \\
\hline 5 & 0.063 & 1.4 & 99.4 & 0.243 & 0.916 & 15.995 & 16 & 0.453 \\
\hline 6 & 0.028 & 0.6 & 100 & 0.164 & 0.973 & 4.947 & 7 & 0.666 \\
\hline
\end{tabular}


Table.5 Canonical discriminant coefficient showing the contribution of each character for the variation

\begin{tabular}{|l|l|l|}
\hline & 1 & 2 \\
\hline DFF & 0.269 & -0.022 \\
\hline LL $(\mathrm{cm})$ & 0.093 & 0.028 \\
\hline PH $(\mathrm{cm})$ & 0.928 & -0.228 \\
\hline NPT & 0.023 & -0.117 \\
\hline PL $(\mathrm{cm})$ & 0.035 & 0.39 \\
\hline DM & -0.139 & 0.079 \\
\hline NFG & -0.245 & 0.744 \\
\hline SF $(\%)$ & 0.188 & 0.344 \\
\hline GL $(\mathrm{mm})$ & 0.078 & -0.281 \\
\hline GW $(\mathrm{mm})$ & -0.158 & 0.194 \\
\hline GLWR & -0.155 & 0.094 \\
\hline SPY $(\mathrm{g})$ & 0.032 & 0.133 \\
\hline canonical correlation & 0.851 & 0.748 \\
\hline level of significance & 0.01 & 0.01 \\
\hline Variance accounted for & 58.4 & 28.2 \\
\hline Cumulative variance & 58.4 & 86.7 \\
\hline
\end{tabular}

Multivariate analysis i.e. UPGMA cluster analysis, principal component analysis and canonical Discriminant analysis used to study genetic variability has revealed wide genetic variation among rice germplasm accessions. According to Aliyu et al., (2000) cluster analysis has the singular efficacy and ability to identify crop accessions with highest level of similarity using the dendrogram. Cluster analysis has revealed seven groups and the genotypes were distributed across all the clusters. Though origin has significant role in clustering i.e. most of the landraces from Tamil Nadu has been confined to cluster 2, > $50 \%$ of exotic lines has assigned to cluster 4 , phenology has played prominent role in clustering along with morphological and grain traits. Cluster 1 has two landraces RG1 (mapillai samba) and RG 106 (Katta samba) which are the late maturing genotypes and duration of 50\% flowering was123 and 119 respectively. Genotypes with early 50\% flowering duration and maturity period have been grouped in cluster 6 (RG69, RG111,
RG104). These genotypes have short stature and lesser leaf length. Cluster 7 has one landrace RG164 (Thillainayagam) which has higher single plant yield, medium height, lengthier leaf and panicles. Cluster 5 has 6 genotypes (RG24, RG25, RG44, RG46, RG54 and RG92) which are taller plant types with less spikelet fertility and higher leaf length. Cluster 3 has 21 genotypes that are having lesser number of filled grains per panicle, spikelet fertility and single plant yield as it has clustered mainly based on grain (yield) traits. The second largest cluster 4 has 62 genotypes with half of the exotic lines has medium grain length, width and grain length width ratio and it has short to medium stature genotypes. The largest cluster 2 with 97 genotypes is dominated by landraces. It is characterized with the genotypes of median performance for all measured traits.

Similar type of clustering based on phenology and morphological characteristics was reported by Sanni et al., (2012) in 434 
landraces of rice collected from African continent. In his study, the late maturing landraces has clustered in 4, 5 and 6 clusters and early maturing accessions in cluster 2 and the total number of tillers was lowest in cluster 7 and cluster 3 has classified based on grain characteristics. Clustering analysis in coconut by Odewale et al., (2012), tall cultivars were grouped in one cluster and dwarf cultivars in another cluster. Worede et al., (2014) has also reported clustering based on flowering, plant type and yield traits in 24 rice genotypes for 17 traits.

Principal Component Analysis measures the importance and contribution of each component to total variance. It can be used for measurement of independent impact of a particular trait to the total variance whereas each coefficient of proper vectors indicates the degree of contribution of every original variable with which each principal component is associated. The higher the coefficients, regardless of the sign, the more effective they will be in discriminating between accessions. There are no inferential tests to prove significance of proper values and the coefficients (Sanni et al., 2012). The current study was based on the Proportion of Variance Criterion by O'Rourke and Hatcher (2013). According to this criterion, Five principal components with cumulative variance of $80.6 \%$ was extracted which gives the clear idea of structure underlying the variables analyzed.

Total variation in each principal axis is determined by number of variables. In the current study, Component 1 has the contribution from Days to $50 \%$ flowering, leaf length, plant height, panicle length, days to maturity and number of filled grains for $28.46 \%$ of the total variability. The first component has phenological and yield related variables. Similar type of performance was obtained by Sanni et al., (2012) and Sanni et al., (2008). Guei et al., (2005) has obtained similar pattern for phenological variables in rice

Takeda (1990) reported that grain size that may be indicated by weight, volume, or length is one of the most important agronomic traits in rice. In current study also, grain width and grain length width ratio has contributed $16.8 \%$ of total variability in component 2 . The remaining variability of $14.4 \%, 11.7 \%$ and $9.3 \%$ was consolidated in component 3 , component 4 and component 5 by various traits such as spikelet fertility, single plant yield, grain length and number of productive tillers.. Rai et al., (2013) has also reported similar results that grain characteristics along with panicle density, leaf length and plant height contributes for phenotypic diversity in a study involving Indian landraces of aromatic and non-aromatic accessions. Caldo et al., (1996) has reported similar results that the traits such as maturity, heading, plant height, culm length, leaf length, and tillering ability were found to be the major factors contributing to the variation of parental lines of modern Philippine rice cultivars. Thus, the prominent characters coming together in a particular principal component by contributing towards variability has the tendency to hang together offer opportunity for its utilization in crop breeding (Chakravorty et al., 2013).

Dissimilarity estimated by multivariate criterion is useful to determine the traits causing the dissimilarity to arise and the relative contributions of various characters to the total variability in the germplasm (Ariyo, 1993). The canonical Discriminant analysis in current study has shown that the days to $50 \%$ flowering, maturity, grain characters, panicle length and plant height contributes for the total variability which is consistent with the results obtained by Sanni et al., (2012) as the first and second canonical discriminant function is dominated by the loadings from number of days to $50 \%$ heading and maturity, 
grain width, plant height, tiller number, and grain length.

Principal component analysis and canonical vector analysis has identified few characters that plays prominent role in classifying the variation existing in the germplasm set. Both the analysis identified days to $50 \%$ flowering, days to maturity plant height, number of filled grains, spikelet fertility, panicle length and grain length in different principal components and vectors are the most important for classifying the variation. Thus the results of principal component analysis and canonical vector analysis confirmed the result of cluster analysis. Sanni et al., (2008, 2012) has also reported similar results with these multivariate analyses. Hence the three multivariate techniques used in the study has revealed the high level of genetic variation existing in the population panel and explains the traits contributing for this diversity.

As rice has enormous genetic diversity, its utilization primarily depends on the way of identifying variation in a population. Multivariate analysis employed in this study has clearly depicted the diversity and contributing characters of the population. Hence the results will be of greater benefit to identify parents for improving various morphological traits analyzed in this study.

\section{References}

Aliyu, B, Ng, NQ, and Fawole, I. (2000). Inheritance of Pubescence in crosses between Vigna unguiculata and $\mathrm{V}$. rhomboidea. Nig. J. Genet, 15, 9-14.

Ariyo, OJ. (1993). Genetic diversity in West African okra (Abelmoschus caillei)(A. Chev.) Stevels-Multivariate analysis of morphological and agronomic characteristics. Genetic Resources and Crop Evolution, 40(1), 25-32.

Ariyo, QJ, and Odulaja, A. (1991). Numerical analysis of variation among accessions of Okra [Abelmoschus esculentus (L.) Moench], Malvaceae. Annals of Botany, 67(6), 527-531.

Caldo, RA, Sebastian, LS, and Hernandez, JE. (1996). Morphology-based genetic diversity analysis of ancestral lines of Philippine rice cultivars. Philippine Journal of Crop Science, 21(3), 86-92.

Chakravorty, Ashim, Ghosh, PD, and Sahu, PK. (2013). Multivariate analysis of phenotypic diversity of landraces of rice of West Bengal. American Journal of Experimental Agriculture, 3(1), 110123.

Committee, IBPGR-IRRI Rice Advisory, and Resources, International Board for Plant Genetic. (1980). Descriptors for rice, Oryza sativa L: Int. Rice Res. Inst.

Firoz, MMD, Golan, MD, Abdur, R (2008). Genetic diversity analysis in some advanced lines of Brassica napus. Science Asia 34: 432-434.

Ghaderi, A, Shishegar, M, Regai, A, Ehdiae, B (1979). Multivariate analysis of genetic diversity for yield and its components in Mungbean. J, Am. Soc. Hort. Sci. 104: 7 28-32.

Guei, R.G., K.A. Sanni, F.J. Abamu, and I. Fawole. 2005. Genetic diversity of rice (Oryza sativa (L.). Agron. Africaine 5:17-28.

Hatcher, Larry, and O'Rourke, Norm. (2014). A step-by-step approach to using SAS for factor analysis and structural equation modeling: Sas Institute.

Kaufman, Leonard, and Rousseeuw, Peter J. (2009). Finding groups in data: an introduction to cluster analysis (Vol. 344): John Wiley and Sons.

Liakat Ali, M, McClung, Anna M, Jia, Melissa H, Kimball, Jennifar A, McCouch, Susan R, and Georgia, C Eizenga. (2011). A rice diversity panel evaluated for genetic and agromorphological diversity between 
subpopulations and its geographic distribution. Crop Science, 51(5), 20212035.

Lu, Kaiyang, Li, Lanzhi, Zheng, Xingfei, Zhang, Zhihong, Mou, Tongmin, and $\mathrm{Hu}$, Zhongli. (2008). Quantitative trait loci controlling $\mathrm{Cu}, \mathrm{Ca}, \mathrm{Zn}, \mathrm{Mn}$ and $\mathrm{Fe}$ content in rice grains. Journal of genetics, 87(3), 305.

Main, M, Bahl, PN (1989). Genetic divergence and hybrid performance in chickpea. Indian J. Genet plant breed. 49: 119-29.

Odewale, JO, Collins, Agho, Ataga, CD, Aisueni, NO, Ikuenobe, CE, Okoye, MN, Uwadiae, EO. (2012). Pattern of genetic diversity and variability in germplasm resources of local and exotic coconut (cocos nucifera 1.) cultivars in Nigeria. Scholarly Journal of Agricultural Science 2(9), 202-207.

Oyelola, BA (2004). The Nigerian Statistical Association preconference workshop, 20-21 September, 2004. Conference centre, University of Ibadan.

Ray, Avik, Deb, Debal, Ray, Rajasri, and Chattopadhayay, Balaji. (2013). Phenotypic characters of rice landraces reveal independent lineages of shortgrain aromatic indica rice. AoB plants, 5 , plt032.

Rezai, A, and Frey, KJ. (1990). Multivariate analysis of variation among wild oat accessions - seed traits. Euphytica, 49(2), 111-119.

Riggs TJ (1973) The use of canonical analysis for selection within a cultivar of spring barley. Ann. Appl. Biol.74: 249-258.

Sanni, KA, Fawole, I, Ogunbayo, A, Tia, D,
Somado, EA, Futakuchi, K, Guei, RG. (2012). Multivariate analysis of diversity of landrace rice germplasm. Crop Science, 52(2), 494-504.

Sanni, Kayode A, Fawole, I, Guei, RG, Ojo, DK, Somado, Eklou A, Tia, D Daniel,... Sanchez, I. (2008). Geographical patterns of phenotypic diversity in Oryza sativa landraces of Côte d'Ivoire. Euphytica, 160(3), 389-400.

Sharma, Bhawana, and Singh, Brijvir. (2012). Canonical Discriminant Analysis for the Assessment of Genetic Variation in Soybean (Glycine max (L.) Merrill). Indian Journal of Plant Genetic Resources, 25(3), 251-256.

Sneath, Peter HA, and Sokal, Robert R. (1973). Numerical taxonomy. The principles and practice of numerical classification.

Tai GCC (1989) A proposal to improve the efficiency of index selection by 'rounding'. Theor. Appl. Genet.78: 798-800.

Takeda, K. (1991). Inheritance of grain size and its implications for rice breeding. Rice genetics II. IRRI, Manila, 181-189.

Vaylay, Ravi, and van Santen, Edzard. (2002). Application of canonical discriminant analysis for the assessment of genetic variation in tall fescue. Crop Science, 42(2), 534-539.

Worede, Fisseha, Sreewongchai, Tanee, Phumichai, Chalermpol, and Sripichitt, Prapa. (2014). Multivariate Analysis of Genetic Diversity among some Rice Genotypes Using Morpho-agronomic Traits. Journal of Plant Sciences, 9(1).

\section{How to cite this article:}

Vishnu Varthini, N., D. Sudhakar, M. Raveendran, S. Rajeswari, S. Manonmani, Shalini Tannidi, P. Balaji Aravindhan, Govindaraj Ponniah, Karthika Gunasekaran and Robin, S. 2017. Rice Diversity Panel Evaluated for Agro-Morphological Diversity by Multivariate Analysis. Int.J.Curr.Microbiol.App.Sci. 6(11): 3887-3901. doi: https://doi.org/10.20546/ijcmas.2017.611.455 